\title{
No benefit of cerebral perfusion over hypothermia in pulmonary endarterectomy
}

A study by investigators from the UK national center for pulmonary endarterectomy (PEA), has shown that postoperative outcomes for antegrade cerebral perfusion (ACP) during PEA are not superior to those for the morewidely used strategy of deep hypothermic

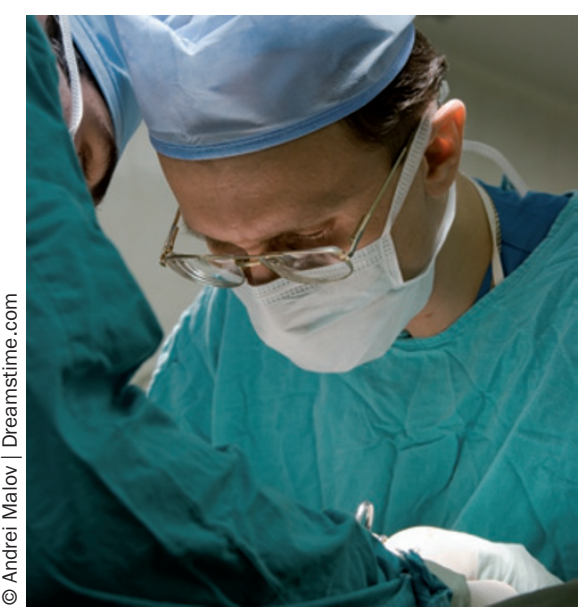

circulatory arrest (DHCA). Importantly, neither approach is associated with postoperative cognitive impairment.

PEA substantially improves survival among patients who develop chronic thromboembolic pulmonary hypertension after pulmonary embolism. DHCA provides the complete cessation of blood flow needed to perform the surgery successfully. "DHCA has been used for many years, but many teams have tried to find ways to keep the brain perfused, in the belief that maintaining blood circulation through the brain would be better," explains lead author of the Lancet report, Alain Vuylsteke. However, "intensivists ... could not relate the postoperative recovery to the type of surgical technique," he continues. "Perfusing the brain ... did not appear to make a difference clinically." Moreover, critics have commented that ACP compromises the surgical field, thus preventing complete endarterectomy. Vuylsteke et al. have conducted the first randomized trial comparing the two approaches.

At 12 and 52 weeks after surgery, cognitive function had improved in both the ACP and DHCA groups, with no between-group differences. Moreover, postoperative hemodynamic improvement, NYHA functional class, and quality of life were comparable between the two groups. "We have modified patient care as a result of the trial and are using DHCA," concludes Dr Vuylsteke. The results of the study also have implications for other procedures that require complete cessation of blood flow, such as aortic surgery.

\section{Alexandra King}

Original article Vuylsteke, A. et al. Circulatory arrest versus cerebral perfusion during pulmonary endarterectomy (PEACOG): a randomised controlled trial. Lancet 378 , 1379-1387 (2011) 